\title{
Comparing the Intensities and Spectral Resolution Achieved by Wavelength- Dispersive Spectrometers on Electron Microprobes and SEMs.
}

Stephen M. Seddio ${ }^{1}$ and John H. Fournelle ${ }^{2}$

1. Thermo Fisher Scientific, Fitchburg, WI, USA.

2. Department of Geoscience, University of Wisconsin-Madison, Madison, WI, USA.

EMPs (electron microprobes) use WD (wavelength-dispersive) spectrometers that rely on curved diffractors and Rowland circle geometry. Today, EMPs are outfitted with as many as five WD spectrometers. Individual WD spectrometers are also available for SEMs. These spectrometers rely on either Rowland circle geometry or parallel beam geometry (hereafter, PB-WDS). The latter was developed to improve X-ray count rates in order to counter the generally lower beam current in an SEM relative to an EMP. The improved count rate in PB-WDS is the result of a much larger solid angle as a collimating optic is inserted deep into the SEM sample chamber during sample analysis. This study compares the X-ray intensities and spectral resolution achievable by the Rowland circle geometer, as mounted on an EMP and the PB-WDS geometry as singly mounted on an SEM.

EMP measurements were made using the Cameca SX51 at the University of Wisconsin-Madison, which has five $160 \mathrm{~mm}$ diameter Rowland circle wavelength-dispersive spectrometers, three of which contain low-pressure P10 flow-though detectors and two of which contain high-pressure P10 flow-though detectors. SEM measurements were made using a Thermo Scientific ${ }^{\mathrm{TM}}$ MagnaRay ${ }^{\mathrm{TM}}$ PB-WDS, which contains a sealed Xe detector, mounted on a JEOL 7001F FE-SEM. Measurements were made under similar or, when possible, identical conditions (e.g., accelerating voltage, beam current, count times, and diffractor) on the same metal standards. In order to compare intensities across a wide range of wavelengths, X-ray intensities were measured on $\mathrm{B} \mathrm{K} \alpha, \mathrm{C} \mathrm{K} \alpha, \mathrm{Al} \mathrm{K} \alpha$, Si $\mathrm{K} \alpha$, Ti $\mathrm{K} \alpha, \mathrm{Fe} \mathrm{K} \alpha$, and $\mathrm{Cu} \mathrm{K} \alpha$. The diffractors used by the EMP were PC3 $\left(\mathrm{MoB}_{4} \mathrm{C}\right)$ for B; PC2 (NiC95) for B and C; PC1 (WSi60) for $\mathrm{C}$; TAP for $\mathrm{Al}$ and $\mathrm{Si}$; PET for $\mathrm{Si}$ and $\mathrm{Ti}$; $\mathrm{LiF} 200$ for $\mathrm{Ti}, \mathrm{Fe}$, and $\mathrm{Cu}$; and $\mathrm{LiF} 220$ for $\mathrm{Cu}$. When possible, measurements were made concurrently using both high- and low-pressure P10 detectors on multiple spectrometers. If measurements were made on multiple spectrometers using the same diffractors and detector type (i.e., high- or low-pressure Ar), the reported intensities are those of whichever spectrometer yielded higher intensities. The diffractors used by the parallel beam WDS were $\mathrm{MoB}_{4} \mathrm{C}$ for B; NiC80 for B and C; WSi60 for C; TAP for Al and Si; PET for Si and Ti; and LiF200 for Ti, Fe, and $\mathrm{Cu}$. A MagnaRay can concurrently contain six diffractors. The LiF220 diffractor was not available in the MagnaRay used in this study. The reported LiF220 Cu intensity for MagnaRay is the average of the intensities obtained by the factory from all MagnaRays containing LiF220 diffractors. In order to maximize count rates, detectors for both the EMP and the SEM were set to integral mode. Additionally, peak searches were done to assure that the spectrometers were positioned correctly to maximize count rates for a given element.

Results are summarized in Figure 1 and Table 1.The PB-WDS yields dramatically higher intensities relative to the EMP for low-energy X-rays (e.g., B, C). The EMP generated higher intensities than the PB-WDS for Al and Si when measured on TAP. For higher energy X-rays (e.g., Ti, Fe, Cu), PB-WDS typically yields higher intensities compared to the low-pressure detectors of the EMP but lower intensities compared to the high-pressure detectors of the EMP. Figure 2 shows LiF wavescans from the MagnaRay (red) and EMP (blue). 


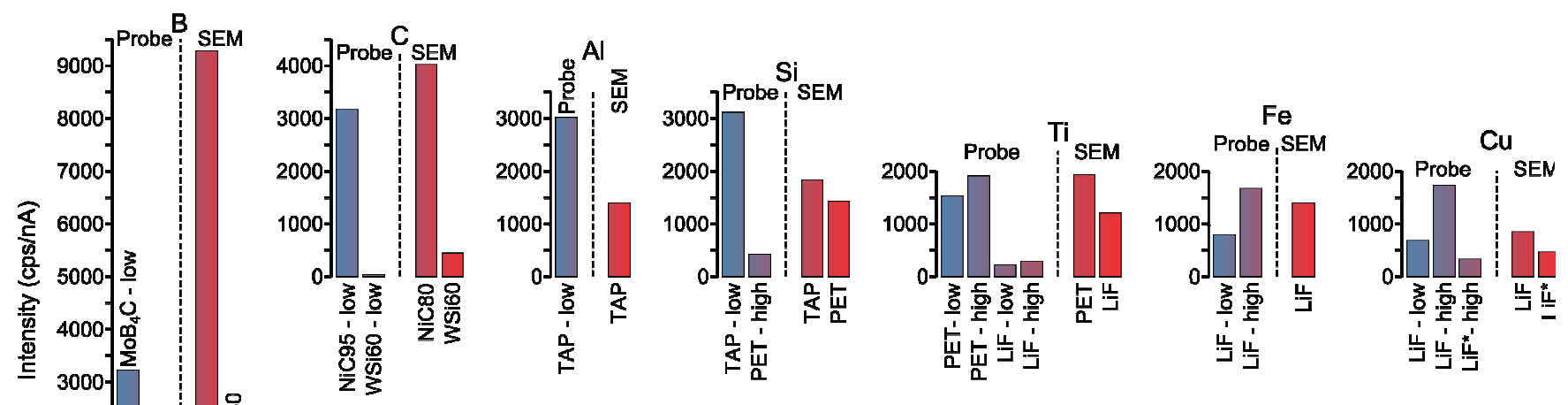

Figure 1. Measured intensities for selected elements. Diffractor information is included. For EMP measurements, "low" and "high" refer to whether the intensities were measured with a low- or high-pressure detector. "LiF*" refers to a $\mathrm{LiF} 220$ diffractor.

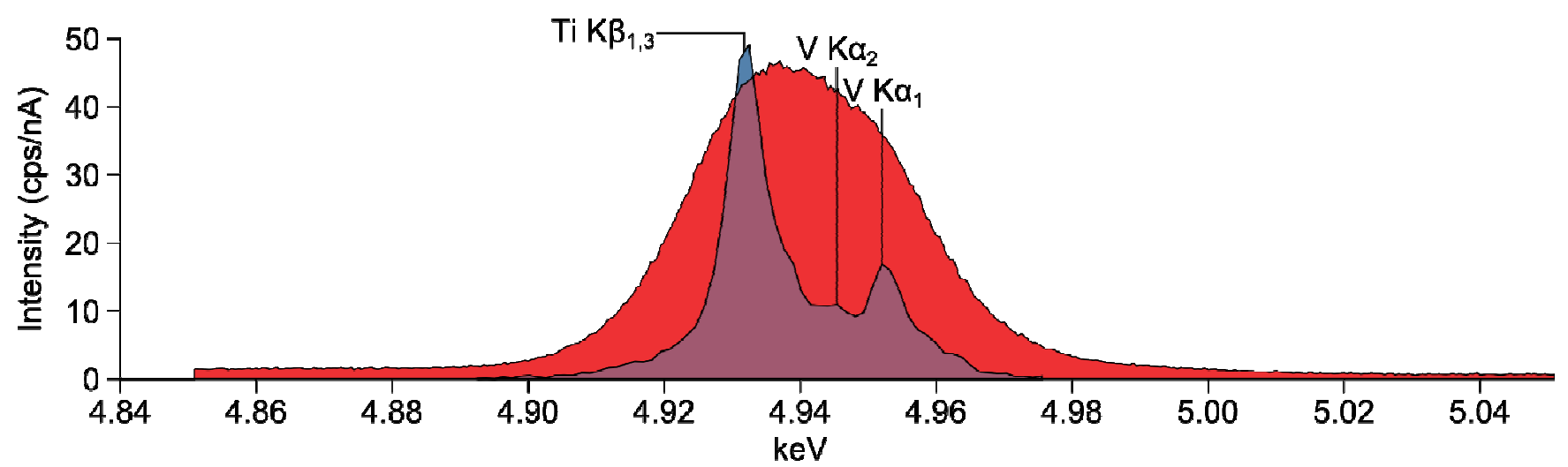

Figure 2. LiF wavescans of the Ti $\mathrm{K} \beta$ and $\mathrm{V} \mathrm{K} \alpha$ spectral region of a Ti-6Al-4V alloy. Microprobe data is blue. MagnaRay data is red. The overlap of the spectra is presented as purple for clarity.

Table 1. WDS intensities measured using the EMP and SEM

\begin{tabular}{|c|c|c|c|c|c|c|c|c|c|c|c|c|}
\hline & \multicolumn{6}{|c|}{ SX51 } & \multicolumn{6}{|c|}{ MagnaRay } \\
\hline & Diff. & Det. & $\mathbf{k V}$ & $\mathbf{N}$ & Ave. & $\boldsymbol{\sigma}$ & Diff. & Det. & $\mathbf{k V}$ & $\mathbf{N}$ & Ave. & $\sigma$ \\
\hline \multirow{2}{*}{ В Ka } & $\mathrm{MoB}_{4} \mathrm{C}$ & P10, low-P & 10 & 6 & 3218.7 & 27.8 & $\mathrm{MoB}_{4} \mathrm{C}$ & \multirow{2}{*}{$\mathrm{Xe}$} & \multirow{2}{*}{10} & \multirow{2}{*}{9} & 9124.1 & 216.2 \\
\hline & \begin{tabular}{|l|}
$\mathrm{NiC} 95$ \\
\end{tabular} & P10, low-P & 10 & 5 & 384.0 & 11.1 & $\mathrm{NiC} 80$ & & & & 1619.3 & 43.4 \\
\hline \multirow{2}{*}{ C Ka } & NiC95 & \multirow{2}{*}{ P10, low-P } & \multirow{2}{*}{10} & \multirow{2}{*}{7} & 3177.9 & 34.4 & $\mathrm{NiC} 80$ & \multirow{2}{*}{$\mathrm{Xe}$} & \multirow{2}{*}{10} & \multirow{2}{*}{24} & 4022.9 & 68.6 \\
\hline & WSi60 & & & & 34.3 & 1.58 & WSi60 & & & & 440.4 & 8.66 \\
\hline Al Ka & TAP & P10, low-P & 20 & 8 & 3014.6 & 47.9 & TAP & $\mathrm{Xe}$ & 20 & 25 & 1390.2 & 15.3 \\
\hline \multirow{2}{*}{ Si Ka } & TAP & P10, low-P & \multirow{2}{*}{20} & 10 & 3112.3 & 3.98 & TAP & \multirow{2}{*}{$\mathrm{Xe}$} & \multirow{2}{*}{20} & \multirow{2}{*}{14} & 1835.9 & 8.61 \\
\hline & PET & P10, high-P & & 5 & 413.2 & 1.18 & PET & & & & 1418.4 & 17.7 \\
\hline \multirow{4}{*}{ Ti Ka } & \multirow{2}{*}{ PET } & P10, low-P & \multirow{4}{*}{20} & \multirow{4}{*}{7} & 1530.2 & 4.20 & PET & $\mathrm{Xe}$ & 20 & 13 & 1930.6 & 13.9 \\
\hline & & P10, high-P & & & 1903.9 & 4.26 & & & & & & \\
\hline & \multirow{2}{*}{$\mathrm{LiF}$} & P10, low-P & & & 218.9 & 0.84 & $\mathrm{LiF}$ & $\mathrm{Xe}$ & 20 & 13 & 612.8 & 9.49 \\
\hline & & P10, high-P & & & 291.4 & 1.68 & & & & & & \\
\hline \multirow{3}{*}{ Fe Ka } & \multirow{2}{*}{$\mathrm{LiF}$} & P10, low-P & \multirow{3}{*}{30} & \multirow{3}{*}{8} & 795.2 & 2.15 & $\mathrm{LiF}$ & $\mathrm{Xe}$ & 30 & 9 & 1164.3 & 5.32 \\
\hline & & \multirow{2}{*}{ P10, high-P } & & & 1668.9 & 1.85 & & & & & & \\
\hline & LiF220 & & & & 544.8 & 2.71 & & & & & & \\
\hline \multirow{3}{*}{$\mathrm{Cu} \mathrm{Ka}$} & \multirow{2}{*}{$\mathrm{LiF}$} & P10, low-P & \multirow{3}{*}{30} & \multirow{3}{*}{4} & 677.2 & 2.35 & $\mathrm{LiF}$ & $\mathrm{Xe}$ & 30 & 8 & 848.6 & 7.58 \\
\hline & & \multirow{2}{*}{ P10, high-P } & & & 1731.6 & 7.32 & & & & & & \\
\hline & LiF220 & & & & 331.3 & 1.60 & & & & & & \\
\hline
\end{tabular}

"Diff." refers to "diffractor." "Det." refers to "detector." "kV" refers to "accelerating voltage". "N" refers to the number of measurements averaged. "Ave." refers to the average of the measured intensities. " $\sigma$ " is the standard deviation of the measured intensities. 